\title{
乌ु
}

\section{Entropy Exchange in a Mixture of Ultracold Atoms}

\author{
J. Catani, ${ }^{1,2}$ G. Barontini, ${ }^{1}$ G. Lamporesi,${ }^{1}$ F. Rabatti, ${ }^{1}$ G. Thalhammer, ${ }^{1}$ F. Minardi,${ }^{1,2}$ S. Stringari, ${ }^{3}$ and M. Inguscio ${ }^{1,2}$ \\ ${ }^{1}$ LENS_European Laboratory for Non-Linear Spectroscopy and Dipartimento di Fisica, Università di Firenze, \\ via N. Carrara 1, I-50019 Sesto Fiorentino-Firenze, Italy \\ ${ }^{2}$ CNR-INFM, via G. Sansone 1, I-50019 Sesto Fiorentino-Firenze, Italy \\ ${ }^{3}$ Dipartimento di Fisica, Università di Trento and CNR-INFM BEC Center, I-38050 Povo, Trento, Italy \\ (Received 11 June 2009; revised manuscript received 16 July 2009; published 28 September 2009)
}

\begin{abstract}
We investigate experimentally the entropy transfer between two distinguishable atomic quantum gases at ultralow temperatures. Exploiting a species-selective trapping potential, we are able to control the entropy of one target gas in presence of a second auxiliary gas. With this method, we drive the target gas into the degenerate regime in conditions of controlled temperature by transferring entropy to the auxiliary gas. We envision that our method could be useful both to achieve the low entropies required to realize new quantum phases and to measure the temperature of atoms in deep optical lattices. We verified the thermalization of the two species in a 1D lattice.
\end{abstract}

DOI: 10.1103/PhysRevLett.103.140401

PACS numbers: 05.30.Jp, 03.75.Hh, 67.85.Pq

In recent years, an intense research of quantum phases common to condensed matter systems and atomic quantum gases has made remarkable progresses [1]. Some of these phases can only be reached provided that the temperature is suitably low. However, in strongly correlated quantum systems, even the temperature measurement can be a challenging task. If so, to ascertain whether a given quantum phase is accessible, it is convenient to focus on the critical value of entropy, rather than temperature. The advantage is especially clear when the strongly correlated regime is reached by sufficiently slow, entropy-preserving, transformations of the trapping potential, as it is often the case for atoms in deep optical lattices [2]. For these reasons, it is important to determine and grasp control of the entropy of degenerate quantum gases [3-5]. In this work, we demonstrate the reversible and controlled transfer of entropy between the two ultracold, harmonically trapped Bose gases, which is based on the use of a species-selective dipole potential (SSDP), i.e., an optical potential experienced exclusively by one species (Fig. 1) [6,7]. In particular, we drive the target gas across the threshold for BoseEinstein condensation, by a reversible transfer of entropy to the auxiliary gas.

The main idea can be understood from textbook thermodynamics. Let us consider two distinguishable gases filling an isolated box, exchanging neither particles nor energy with the outside, and imagine that only one gas (target) is compressed, e.g., through a piston permeable to the second gas (auxiliary). The temperature will increase and, in thermal equilibrium, heat, hence entropy, will transfer from the target to the auxiliary uncompressed gas. In the limit of the auxiliary gas containing a large number of particles, it stands as a thermal bath. In a more formal way, for an ideal gas of $N$ particles, the entropy $S$ is proportional to $N \log (\Sigma / N)$, where the number of accessible singleparticle states $\Sigma$ increases with the energy density of states and with the average energy, i.e., the temperature. In an adiabatic compression of one single gas, the reduction of the energy density of states is compensated by a temperature raising such that $\Sigma$, hence $S$, remains constant. If we add the uncompressed auxiliary gas in thermal contact, the temperature raising must be lower: $\Sigma$ decreases for the target gas (and increases for the auxiliary component). In our experiment, the gases are trapped by adjustable harmonic potentials, but the underlying physics is the same.

To make quantitative predictions, we start from the entropy of an ideal gas at temperature $T$ in a harmonic potential with angular frequency $\omega$ [8]: $S=$ $k_{B} N_{\text {th }}\left[4 g_{4}(z) / g_{3}(z)-\log (z)\right]$, where $N_{\text {th }}$ denotes the number of thermal atoms and the polylogarithmic functions are defined as $g_{n}(z)=\sum_{k \geq 1} z^{k} / k^{n}$. Above the BEC critical temperature $T_{c}, N_{\text {th }}$ equals the total atom number $N$, and the fugacity $z$ is implicitly given by the relation $N=$ $g_{3}(z)\left(k_{B} T / \hbar \omega\right)^{3}$. Below $T_{c}, z=1$ and only the thermal atoms contribute to the entropy, each with a quantity equal to $4 k_{B} \zeta(4) / \zeta(3), \zeta(n)=g_{n}(1)$, so that

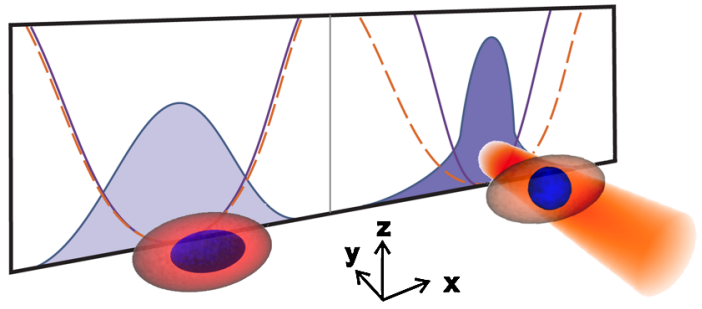

FIG. 1 (color online). Schematic of our experimental procedure. Left: the harmonic magnetic potential is common to both gases, auxiliary (red, larger) and target (blue, smaller). Right: the species-selective dipole beam compresses the target sample and drives it into the degenerate regime. Trapping potentials for the auxiliary $\mathrm{Rb}$ (dashed line) and the target $\mathrm{K}$ gas (solid line) are sketched on the background panels together with the K density distributions. 


$$
S=4 N k_{B} \frac{\zeta(4)}{\zeta(3)}\left(\frac{T}{T_{c}}\right)^{3}=4 k_{B} \zeta(4)\left(\frac{k_{B} T}{\hbar \omega}\right)^{3} .
$$

Thus, below $T_{c}$, the entropy is independent of the total number of atoms $N$.

The above results refer to a noninteracting Bose gas. For high atomic densities, the interatomic interactions modify low energy spectrum and must be taken into account: a theoretical analysis is given in Ref. [9]. We evaluate numerically the implicit expression contained therein and find that, in our experimental circumstances, the interactions increase the ideal gas values of entropy by at most $20 \%$ for condensate samples. An expression for the entropy, easy-to-use and more faithfully approximating the results of [9] than Eq. (1), is obtained by replacing $\left(T / T_{c}\right)^{3}$ in Eq. (1) with the thermal fraction $1-f_{c}$ with $f_{c}$ taken from a semi-ideal model $[9,10]$

$$
f_{c}=1-t^{3}-\eta[\zeta(2) / \zeta(3)] t^{2}\left(1-t^{3}\right)^{2 / 5} .
$$

Here, $t=T / T_{c}, \eta=\mu / k_{B} T$ and the zero-temperature chemical potential $\mu=(\hbar \omega / 2)\left(15 N a_{s} / a_{h o}\right)^{2 / 5}$ depends on $a_{s}$, the scattering length, and $a_{h o}=\sqrt{\hbar / m \omega}$. In our experimental conditions, $\eta$ ranges from 0.30 to 0.33 for condensed samples. This approximation is especially helpful for high condensate fractions $\left(f_{c}>0.5\right)$, where the ideal gas formula Eq. (1) seriously underestimates the gas entropy. However, in the explored range $f_{c}<0.3$, Eq. (1) stands as a reasonably good approximation. For thermal samples above the BEC threshold, the interaction energies are negligible with respect to the temperature. This is also the case for interspecies interactions, since the auxiliary sample, always thermal for the reported data, has low density. For this reason, we simply add the singlespecies contributions to obtain the total entropy of the mixture, $S=S_{\mathrm{K}}+S_{\mathrm{Rb}}$.

In our experiment, we load a mixture of ${ }^{87} \mathrm{Rb}$ and ${ }^{41} \mathrm{~K}$ in a millimetric magnetic trap and sympathetically cool the mixture [11]. The magnetic trap provides a harmonic confinement with frequencies $\left(\omega_{x}, \omega_{y}, \omega_{z}\right)=2 \pi \times$ $(24,297,297) \mathrm{Hz}$ for $\mathrm{K}$ and a factor 1.46 smaller for $\mathrm{Rb}$. The geometric mean frequencies $\omega=\left(\omega_{x} \omega_{y} \omega_{z}\right)^{1 / 3}$ equal to, and we have $\left(\omega_{\mathrm{Rb}}, \omega_{\mathrm{K}}\right)=2 \pi \times(88,128) \mathrm{Hz}$. To selectively act on the potential experienced by the $\mathrm{K}$ atoms (target gas) alone, we use a laser beam tuned to an intermediate wavelength between the $D_{1}$ and $D_{2}$ lines of $\mathrm{Rb}$ (auxiliary gas), such that the dipole forces on $\mathrm{Rb}$ due to these two transitions cancel out [7]. The beam, linearly polarized, with a waist of $55 \mu \mathrm{m}$, propagates along the horizontal $y$ direction (Fig. 1). We experimentally determine the wavelength value to be $789.85(1) \mathrm{nm}$, by minimizing the efficiency of the Raman-Nath diffraction caused by a pulsed standing wave on a $\mathrm{Rb}$ condensate [12]. We also measured the residual potential acting on $\mathrm{Rb}$ to be $V_{\mathrm{Rb}} / V_{\mathrm{K}}=0.08(1)$. Such a residual potential weakly deforms only a small central region of the $\mathrm{Rb}$ density. From the measured temperature increase caused by the SSDP on $\mathrm{Rb}$ alone, we estimate a residual effective increase of $\omega_{\mathrm{Rb}}$ lower than 7\%, hereafter neglected.

On the K sample, the additional confinement induced by the SSDP beam is instead a harmonic potential whose frequencies add in quadrature to those of the magnetic trap. The compression occurs mainly along the weak $x$ axis of the magnetic trap $\left(\omega_{x}\right.$ increases up to a factor $5)$, slightly along the $z$ direction $\left(\omega_{z}\right.$ increases less than $8 \%$ ), and is utterly negligible along the propagation $y$ axis of the SSDP beam. Overall, the dipole beam raises the $\omega_{\mathrm{K}}$, hence the $\mathrm{K}$ critical temperature, up to a factor 1.7.

We raise the power of the SSDP beam with an exponential ramp lasting $200 \mathrm{~ms}$ for maximum compression of the trap frequency $\omega_{\mathrm{K}, f}=2 \pi \times 216 \mathrm{~Hz}$. The adiabaticity is preserved since the ramp is longer than the trapping periods of ${ }^{41} \mathrm{~K}$, i.e., $(41,3.4,3.4) \mathrm{ms}$. Thermal equilibrium between the two gases is maintained throughout by making all transformations slow with respect to the interspecies collision times, a few ms in typical experimental conditions: we verified that the temperatures of the two species are always equal, within our statistical uncertainty. Our observables are the number of thermal atoms $N_{\text {th }}$, the number of condensed atoms $N_{c}$, and the temperature $T$ of the two species that are measured by resonant absorption imaging after all confining potential are removed and the atomic clouds have expanded. For each species, the entropy is either computed from the measured temperature and atom number for samples above BEC or obtained from the condensate fraction below BEC.

In Fig. 2, we show that, upon selective compression, the $\mathrm{K}$ sample crosses the BEC threshold; thus, its entropy manifestly drops. Starting with $9.5 \times 10^{5} \mathrm{Rb}$ atoms and $2 \times 10^{5} \mathrm{~K}$ atoms at $0.410(15) \mu \mathrm{K}$, we measure the $\mathrm{K}$ condensate fraction at different compression ratios. Because of the large size of the SSDP beam compared to the $\mathrm{K}$ cloud, we observe that, in the absence of $\mathrm{Rb}$, the

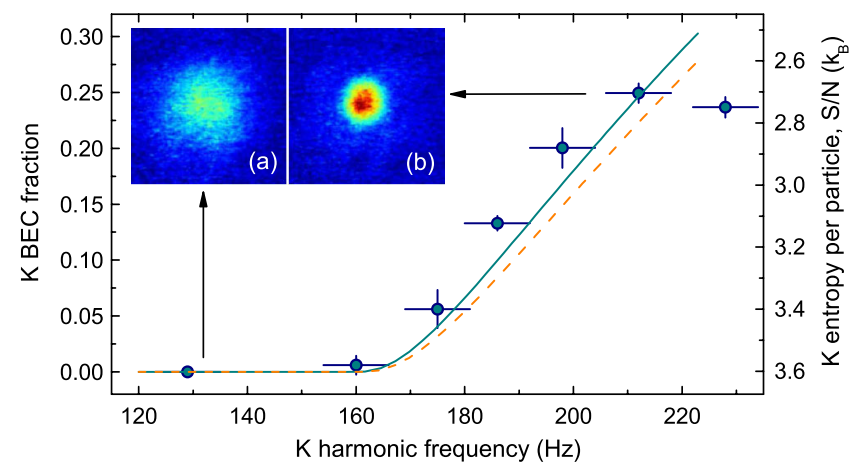

FIG. 2 (color online). BEC fraction of the $\mathrm{K}$ sample as a function of the $\mathrm{K}$ harmonic frequency after the compression. Data (circles) are compared to the theoretical predictions based on the numerical (solid line) and analytical approximate (dashed line) solutions of Eq. (3). On the right axis, the approximated value $S /\left(N k_{B}\right)=4[\zeta(4) / \zeta(3)]\left(1-f_{c}\right)$ is shown. The inset displays absorption images of the $\mathrm{K}$ sample before (a) and after (b) the compression. 
compression does not increase the $\mathrm{K}$ degeneracy, differently from the "dimple" configuration, where a tightly localized dipole trap is used to bring a single species to BEC [13].

For a comparison of the observed condensate fraction after compression with the theoretical predictions, we first calculate the final temperature and then obtain the condensate fraction from Eq. (2). The final temperature $T_{f}$ of the mixture after the transformation $\omega_{\mathrm{K}, i} \rightarrow \omega_{\mathrm{K}, f}$ satisfies the equation:

$$
\begin{aligned}
S\left(T_{i}, \omega_{\mathrm{K}, i}, N_{\mathrm{K}}\right)+S\left(T_{i}, \omega_{\mathrm{Rb}}, N_{\mathrm{Rb}}\right)= & S\left(T_{f}, \omega_{\mathrm{K}, f}, N_{\mathrm{K}}\right) \\
& +S\left(T_{f}, \omega_{\mathrm{Rb}}, N_{\mathrm{Rb}}\right)
\end{aligned}
$$

where $T_{i}$ is the initial temperature. In our experimental circumstances, the transformation starts with a thermal $\mathrm{Rb}$ sample and a $\mathrm{K}$ sample at the BEC threshold or below. Therefore, we solve Eq. (3) numerically for $T_{f}$. We also find an analytical approximate solution for $T_{f}$ [14]:

$$
T_{f}=T_{i}\left(\omega_{\mathrm{K}, f} / \omega_{\mathrm{K}, i}\right)\left\{W\left[\left(\omega_{\mathrm{K}, i} / \omega_{\mathrm{K}, f}\right)^{3} \xi e^{\xi}\right] / \xi\right\}^{1 / 3}
$$

where $\xi=4 \zeta(4)\left(k_{B} T_{i} / \hbar \omega_{\mathrm{K}, i}\right)^{3} / N_{\mathrm{Rb}}$ and $W(y)$ is the Lambert's function defined as the inverse of $y=x e^{x}$. In Fig. 2, we plot the condensate fraction, and the related entropy per particle, as a function of the $\mathrm{K}$ harmonic frequency at the end of the compression. The data points are well in agreement with the numerical results that differ from the simple analytical solution obtained from Eq. (2) and (4) by only $10 \%$ in our range of compressions.

We now illustrate how the interspecies exchange of entropy can be used to explore the entropy-temperature diagram of a sample of $1.1(2) \times 10^{5} \mathrm{~K}$ atoms, shown in Fig. 3. Notice that here we focus on the properties of the target $\mathrm{K}$ gas, but the presence of the auxiliary $\mathrm{Rb}$ gas is essential for some transformations. At each data point, the entropy of the gas is determined in the following way: above $T_{c}$, we compute the entropy per particle by the formula $S / N k_{B}=4-\log \left[N\left(\hbar \omega / k_{B} T\right)^{3}\right]$ obtained by truncating the polylogarithmic functions at the first order in $z$; below $T_{c}$, we calculate the entropy from the measured condensate fraction $S / N k_{B}=4[\zeta(4) / \zeta(3)]\left(1-f_{c}\right)$.

By combining sympathetic cooling and control of the harmonic frequencies, we can follow different trajectories in the $S$ - $T$ diagram. In particular, we compare the efficiency of three trajectories indicated as A, B, C in Fig. 3. Trajectory A corresponds to plain sympathetic cooling in the magnetic trap. Along B, instead, we raise the SSDP beam to maximum power when the temperature of the samples reaches $0.6 \mu \mathrm{K}$, and we proceed with evaporation of $\mathrm{Rb}$ and sympathetic cooling of $\mathrm{K}$. When the $\mathrm{Rb}$ is exhausted, at $0.41 \mu \mathrm{K}$, we extinguish the SSDP beam intensity, thus decompressing the trapping potential, and reach the same final point of the previous trajectory. Finally along $\mathrm{C}$, we raise the SSDP when the $\mathrm{K}$ sample is close to BEC at $0.34 \mu \mathrm{K}$ and cross threshold by selective compres-

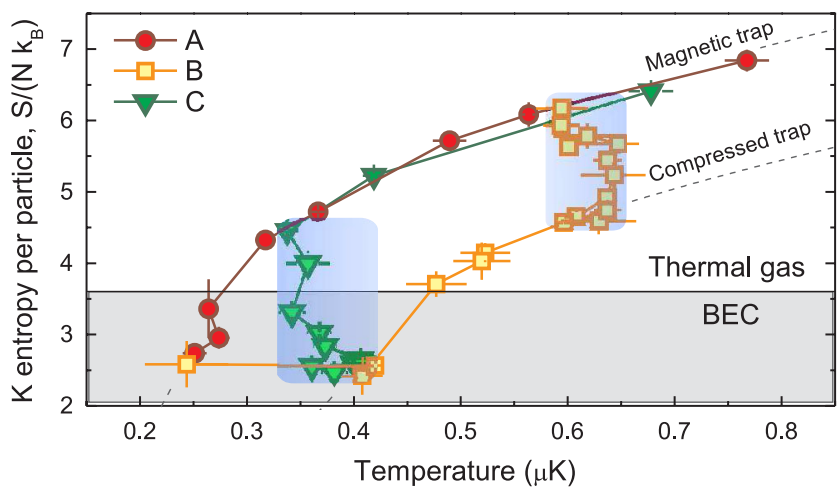

FIG. 3 (color online). Trajectories in the entropy-temperature diagram of the $\mathrm{K}$ sample (see text). For the experimental data, the entropy is determined with the formulas in text. The dashed lines show the ideal entropy for a sample of $1.1 \times 10^{5} \mathrm{~K}$ atoms at the harmonic frequencies of the magnetic trap $\left(\omega_{\mathrm{K}}=\right.$ $2 \pi \times 128 \mathrm{~Hz}$ ) and of the SSDP maximally compressed trap $\left(\omega_{\mathrm{K}}=2 \pi \times 216 \mathrm{~Hz}\right)$. The shaded boxes highlight the isothermal SSDP compressions.

sion as in Fig. 2. The end point of all trajectories is reached when the $\mathrm{Rb}$ sample is either exhausted (B) or depleted to the point that further sympathetic cooling is inefficient, $N_{\mathrm{Rb}} / N_{\mathrm{K}} \sim 2$ (A, C). The three described paths have similar efficiencies, since the end values of entropy are approximately equal. It is important to notice that, for larger $N_{\mathrm{Rb}} / N_{\mathrm{K}}$, all cooling processes are more efficient, and lower $S / N$ values are likely attainable. By means of the SSDP compression in presence of the auxiliary $\mathrm{Rb}$ gas, we perform isothermal transformations. Vice versa, with a single species, an adiabatic variation of the trapping frequencies corresponds to an isoentropic transformation that does not increase the gas degeneracy.

The degree of reversibility of the SSDP transformations across the $\mathrm{K} \mathrm{BEC}$ threshold is investigated by performing multiple compression and decompression cycles. The SSDP beam intensity is repeatedly ramped up and down in an exponential fashion $(\tau=45 \mathrm{~ms})$ with a period of $0.43 \mathrm{~s}$. As shown in Fig. 4, the BEC threshold is crossed for 5 cycles at maximum compression. The $\mathrm{K}$ condensate fraction decreases over successive compressions because, at each cycle, the number of $\mathrm{Rb}$ atoms is reduced and the temperature slightly increased. Even in absence of compression, our $\mathrm{Rb}$ sample experiences a heating rate of the order of $0.7 \mu \mathrm{K} / \mathrm{s}$ which we reduce by an order of magnitude by means of a microwave shield [15], removing trapped $\mathrm{Rb}$ atoms with energy $E / k_{B}>5.5 \mu \mathrm{K}$. As a consequence, the $\mathrm{Rb}$ atom number decreases at a rate of $2.5(5) \times 10^{5} \mathrm{~s}^{-1}$. Starting with approximately $8.5 \times 10^{5}$ $\mathrm{Rb}$ atoms and $2 \times 10^{5} \mathrm{~K}$ atoms at $0.4 \mu \mathrm{K}$, we obtain a finite $\mathrm{K}$ condensate fraction over a time span of $2 \mathrm{~s}$. After 5 cycles, the entropy exchange still occurs, but its efficiency is undermined by the lower $\mathrm{Rb}$ atom number and the temperature before the compression is too high to cross the BEC threshold. Our data can still be described by the theoretical analysis summarized in Eq. (2) and (4), pro- 


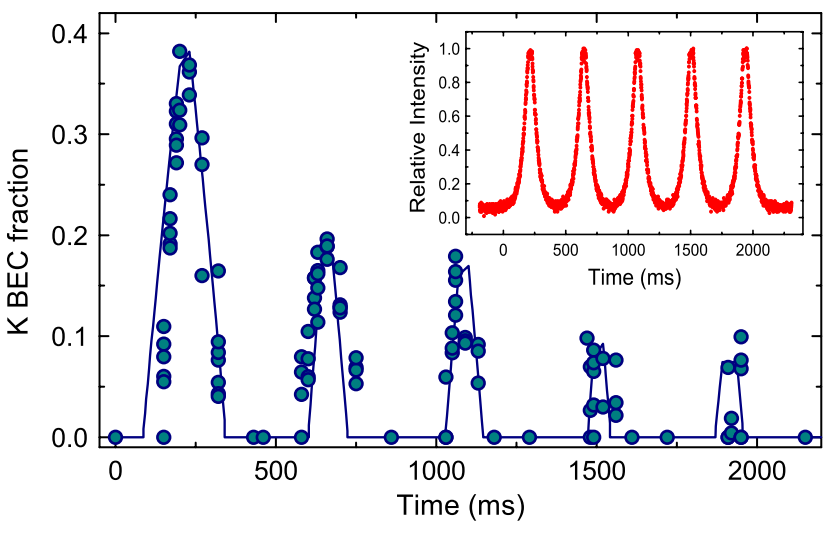

FIG. 4 (color online). Cycles of compressions and decompressions, the $\mathrm{K}$ condensate fraction changes in a reversible manner as we modulate the $\mathrm{K}$ harmonic frequency over time from 128 to $216 \mathrm{~Hz}$ (the solid line guides the eye). The inset shows the relative intensity of the SSDP beam.

vided we take into account that successive cycles occur from different initial conditions.

The independent manipulation of two atomic species opens also new perspectives for the thermometry of cold gases in optical lattices. As a first application, we have investigated the thermalization of the two species when the SSDP forms a 1D lattice for K only. The SSDP standing wave along the $x$ weak axis of the magnetic trap is ramped in $200 \mathrm{~ms}$, kept constant for $20 \mathrm{~ms}$, and then linearly extinguished in $1 \mathrm{~ms}$. The magnetic trap is abruptly switched off immediately afterwards, and the atoms expand freely for $10 \mathrm{~ms}(\mathrm{~K})$ and $15 \mathrm{~ms}(\mathrm{Rb})$. We observe that $\mathrm{Rb} x$ and $y \mathrm{rms}$ sizes do not depend on the SSDP lattice depth and either can be used to extract the gas temperature. We also find that the K temperature obtained from the $y$ size increases with the lattice strength, but it is always equal to $\mathrm{Rb}$ within our statistical uncertainty of $10 \%$. This shows that, in a 1D lattice configuration, up to a strength of 20 recoil energy, the auxiliary gas allows to read out the system temperature and to validate proposed methods to extract the temperature of a gas in an optical lattice [16].

In summary, we have experimentally demonstrated a method to exchange entropy between two gases, whereby we can precisely reduce the entropy of an ultracold atomic sample and drive it across the BEC threshold in a reversible manner. Earlier experiments demonstrating reversible BEC, or simply gain in phase-space density, featured either hydrogen films [17], a single species gas in a "dimple" potential [13] or multiple spin components in the same harmonic potential [18]. Our method is similar in principle to the "dimple" configuration of Ref. [13], where the single gas can be thought as consisting of two components distinguished by their extension. The use of two atomic species combined with the SSDP, however, offers outstanding flexibility and can be easily extended to different configurations, e.g., lattices, and to different mixtures. In particular, the species-selective potential works best for mixtures that combine atoms with widely spaced $D_{1}$ and $\mathrm{D}_{2}$ transitions and largely different resonant wavelengths. Our method can be applied also to reduce the entropy of an ideal degenerate Fermi gas, $S / N=\left(\pi^{2} k_{B}^{2} T\right) /(\hbar \omega \sqrt[3]{6 N})$ for harmonic trapping. In the limit of isothermal transformations, the entropy of the target Fermi gas decreases as the inverse of harmonic frequency compression ratio $S_{f} / S_{i}=$ $\omega_{i} / \omega_{f}$, less than in the case of a condensate $S_{f} / S_{i}=$ $\left(\omega_{i} / \omega_{f}\right)^{3}$.

A species-selective optical lattice has important applications for the thermometry of an atomic Mott insulator, filling the need of convenient experimental techniques [19]. Preliminarily, we have verified thermalization to occur in a 1D SSDP lattice. In addition, the auxiliary component could be used as coolant to dissipate the Mott excitations [20]. Alternatively, dilute atoms localized by a species-selective lattice might be used as disordered scatterers for lattice-insensitive matter waves [21].

This work was supported by MIUR PRIN 2007, Ente CdR in Firenze, CNR under project EuroQUAM DQS, and EU under STREP CHIMONO and NAME-QUAM.

[1] M. Lewenstein et al., Adv. Phys. 56, 243 (2007).

[2] I. Bloch, J. Dalibard, and W. Zwerger, Rev. Mod. Phys. 80, 885 (2008).

[3] M. Popp et al., Phys. Rev. A 74, 013622 (2006).

[4] T.-L. Ho and Q. Zhou, Proc. Natl. Acad. Sci. U.S.A. 106, 6916 (2009).

[5] J.-S. Bernier et al., Phys. Rev. A 79, 061601(R) (2009).

[6] C. Presilla and R. Onofrio, Phys. Rev. Lett. 90, 030404 (2003).

[7] L. J. LeBlanc and J.H. Thywissen, Phys. Rev. A 75, 053612 (2007).

[8] L. Pitaevskii and S. Stringari, Bose-Einstein Condensation (Oxford University Press, Oxford, 2003).

[9] S. Giorgini, L. P. Pitaevskii, and S. Stringari, J. Low Temp. Phys. 109, 309 (1997).

[10] M. Naraschewski and D. M. Stamper-Kurn, Phys. Rev. A 58, 2423 (1998).

[11] L. De Sarlo et al., Phys. Rev. A 75, 022715 (2007).

[12] Y. B. Ovchinnikov et al., Phys. Rev. Lett. 83, 284 (1999).

[13] D. M. D. M. Stamper-Kurn et al., Phys. Rev. Lett. 81, 2194 (1998).

[14] We use $S=4 k_{B} \zeta(4)\left(k_{B} T / \hbar \omega\right)^{3}$ for K condensed samples and truncate all $z$ series to the linear term so that, for $\mathrm{Rb}$ thermal samples, $S=N k_{B}\left(4-\log \left[N\left(\hbar \omega / k_{B} T\right)^{3}\right]\right.$.

[15] M.-O. Mewes et al., Phys. Rev. Lett. 77, 416 (1996).

[16] D. McKay, M. White, and B. DeMarco, Phys. Rev. A 79, 063605 (2009).

[17] P. W. H. Pinkse et al., Phys. Rev. Lett. 78, 990 (1997).

[18] M. M. Erhard et al., Phys. Rev. A 70, 031602(R) (2004).

[19] G. Pupillo, C. J. Williams, and N. V. Prokofev, Phys. Rev. A 73, 013408 (2006).

[20] A. J. Daley, P. O. Fedichev, and P. Zoller, Phys. Rev. A 69, 022306 (2004).

[21] U. Gavish and Y. Castin, Phys. Rev. Lett. 95, 020401 (2005). 